\title{
Linearização por realimentação e aprendizagem por reforço para o controle do sistema de posicionamento do ROV
}

\author{
Lucas S. Cadengue* Samuel A. Fidelis* Gabriel S. Lima* \\ Wallace M. Bessa* \\ * RoboTeAM - Robotics $\&$ Machine Learning Universidade Federal do \\ Rio Grande do Norte, RN, (e-mail: lucassolano@ufrn.edu.br, \\ samico@ufrn.edu.br,limagabriel@ufrn.edu.br,wmbessa@ct.ufrn.br).
}

\begin{abstract}
A ROV (Remotely Operated underwater Vehicle) system needs a precise estimation of its position in order to avoid damage and imprecise movements in certain missions. In this paper, an intelligent controller for the trajectory tracking of a ROV susceptible to external forces (water dynamics, currents, animals, etc.) is proposed based on feedback linearization with an adaptative function optimized using a machine learning strategy. This work proposes an approach to the matter employing an reinforcement learning algorithm, the $\epsilon$-greedy, to let him discover for itself an optimal learning rate for the used compensator. Numerical results confirm a strong improvement in the performance of the controller when the proposed compensator and learning strategy is inserted.

Resumo: Um sistema ROV (Remotely Operated underwater Vehicle) precisa de uma estimação precisa da sua posição para evitar danos e movimentos imprecisos para certas tarefas. Neste artigo, um controlador inteligente foi proposto para o rastreamento da trajetória de um ROV suscetível a forças externas (dinâmica da água, correntes, animais, etc.), este controlador foi baseado no método de linearização por realimentação com uma função adaptativa otimizada utilizando uma estratégia de aprendizagem de máquina. Este trabalho propõe o emprego de um algoritmo de aprendizagem por reforço, o $\epsilon$-greedy, para o deixar descobrir por si só uma taxa de aprendizagem ótima para o compensador. Resultados numéricos confirmam uma forte melhora na performance do controlador quando o compensador proposto e a estratégia de aprendizagem é inserida.
\end{abstract}

Keywords: ROV; intelligent control; feedback linearization; $\epsilon$-greedy; machine learning.

Palavras-chaves: ROV; controle inteligente; linearização por realimentação; $\epsilon$-greedy; aprendizagem de máquina.

\section{INTRODUÇÃO}

ROVs tem sido utilizados em explorações submarinas, em pesquisas de fenômenos subaquáticos e na montagem, inspeção e reparo de estruturas offshore (Ludvigsen and S $\phi$ rensen, 2016; Mindell, 2015; Teague et al., 2018). Os ROVs permitiram substituir o trabalho de pessoas dentro desses veículos por conta dos riscos envolvidos nessa atividade.

$\mathrm{Na}$ execução de certas tarefas com o veículo robótico, o operador precisa monitorar e controlar um certo número de parâmetros. Se alguns desses parâmetros, como por exemplo a posição e a altitude do veículo, puderem ser controlados automaticamente, a teleoperação do ROV pode ser enormemente facilitada (Bessa et al., 2008).

O posicionamento dinâmico de veículos submarinos não tripulados tem sido um estudo recorrente nos artigos mais recentes e confirma a necessidade do desenvolvimento de

^ Os autores agradecem a Coordenação de Aperfeiçoamento de Pessoal de Ensino Superior (CAPES) e ao Conselho Nacional de Desenvolvimento Científico e Tecnológico (CNPq). um controlador que pudesse lidar com a dinâmica inerente do sistema não-linear, coeficientes hidrodinâmicos incertos e perturbações externas (Bessa et al., 2008).

No caso de veículos subaquáticos, as metodologias tradicionais de controle não são a escolha mais adequada e não podem garantir o desempenho e a precisão desejados (Goheen and Jeffreys, 1990; Yuh, 1994).

Para superar este impasse, inteligência computacional pode ser utilizada para estimar os efeitos hidrodinâmicos, como por exemplo, propor um sistema de controle para um agente, utilizando um controlador por modos deslizantes. Esta estratégia consegue aproximar os efeitos provenientes da interação fluido-estrutura, porém esta abordagem tem um grande gasto energético.

O sistema de controle é um dos elementos mais importantes de um veículo robótico subaquático, e suas características (vantagens e desvantagens) desempenham um papel essencial quando se tem que escolher um veículo para uma missão específica (Bessa et al., 2010). A estratégia de controle para posição de um ROV pode ser feita usando 
técnicas tais como Linearização por Realimentação (FB Feedback Linearization), Modos Deslizantes (SMC - Sliding Mode Control) ou PID.

Dentre as técnicas de inteligência computacional, as baseadas na aprendizagem por reforço (RL, do inglês Reinforcement Learning) são interessantes por permitirem que o sistema aprenda por ele mesmo como os efeitos do arrasto hidrodinâmico influenciam no seu próprio comportamento dinâmico. Neste tipo de aprendizagem, temos a realização de um mapeamento entrada-saída através da interação contínua com o ambiente, visando minimizar um índice escalar de desempenho (Haykin, 2007). RL também tem sido incorporada em diversas técnicas de controle para sistemas lineares tanto na forma de compensação de dinâmica não modelada quanto no ajuste dos parâmetros dos controladores utilizados como nos trabalhos (Brinkmann et al., 2018; Kim et al., 2019; Pradeep et al., 2016; Zhu et al., 2015; Jardine et al., 2019; Yang et al., 2018; Bejar and Moran, 2019). A aprendizagem por reforço é um subtópico dentro da aprendizagem de máquinas, porém não se assemelha com a aprendizagem supervisionada e não-supervisionada, sendo considerada o terceiro pilar da aprendizagem de máquinas.

A estratégia de linearização por realimentação tem sido largamente empregada, principalmente na área de robótica industrial, devido à simplicidade na qual ela se apresenta. A ideia por trás do método consiste na escolha de uma lei de controle que permita a transformação do sistema dinâmico original em um sistema dinâmico equivalente, porém mais simples.

Neste trabalho, um controlador FB é proposto para realizar o rastreamento de trajetória de um ROV sujeito a forças hidrodinâmicas de arrasto. Para compensar essas forças externas um compensador adaptativo é adicionado ao controlador.

No RL, o agente aprende através de recompensas (Rewards - R) que são geradas para as ações (Actions - A) que ele toma de acordo com uma política (Policy - П), o objetivo é maximizar as recompensas. Um dos desafios da aprendizagem por reforço é saber escolher entre explorar ou prospectar ações para maximizar sua recompensa, ou seja, o agente teve escolher entre explorar um nova ação ou continuar em uma ação já conhecida por exemplo.

Um dos vários algoritmos de aprendizagem por reforço é o $\epsilon$-greedy.

O algoritmo $\epsilon$-greedy é um método simples de resolver o problema do bandido de vários braços propondo uma forma de balancear o fator de exploração e prospecção do agente.

\section{CONTROLE DE PROFUNDIDADE}

Um modelo adequado capaz de descrever a dinâmica de um ROV deve considerar não só a dinâmica de corpo rígido como também as interações fluídicas com o meio (Bessa et al., 2010). As equações de movimento para veículos subaquáticos podem ser expressas, com respeito ao sistema referencial fixo ao corpo, na seguinte forma:

$$
\boldsymbol{M} \dot{\boldsymbol{v}}+\boldsymbol{k}(\boldsymbol{v})+\boldsymbol{h}(\boldsymbol{v})+\boldsymbol{g}(\boldsymbol{x})=\boldsymbol{u}
$$

onde $\boldsymbol{v}=\left[\begin{array}{llllll}v_{x} & v_{y} & v_{z} & \omega_{x} & \omega_{y} & \omega_{z}\end{array}\right]^{\top}$ é o vetor de velocidades lineares e angulares no referencial móvel, $\boldsymbol{x}=\left[\begin{array}{lllll}x & y & z & \alpha & \beta\end{array}\right]^{\top}$ representa a posição e orientação com respeito ao referencial inercial, $\boldsymbol{M}$ é a matriz de inércia, $\boldsymbol{k}(\boldsymbol{v})$ é o vetor de forças centrífugas e de Coriolis, $\boldsymbol{h}(\boldsymbol{v})$ representa os efeitos hidrodinâmicos, $\boldsymbol{g}(\boldsymbol{x})$ ) é o vetor de forças restauradoras (gravidade e flutuabilidade), e $\boldsymbol{u}$ é o vetor de forças e momentos.

No caso particular ROVs, a maioria dos veículos são projetados de modo que os ângulos de roll $(\alpha)$ e de pitch $(\beta)$ sejam estabilizados passivamente. Isso permite simplificar o modelo do sistema para quatro graus de liberdade, $\boldsymbol{x}=\left[\begin{array}{llll}x & y & z & \gamma\end{array}\right]^{\top}$, permitindo assim um desacoplamento do movimento na direção vertical $(z)$ e no plano $X Y$ (variáveis $x, y$ e $\gamma$ ) (Bessa et al., 2010).

O movimento vertical do ROV pode ser escrito com base na equação (Bessa et al., 2008):

$$
m \ddot{z}=u+h
$$

onde $m$ é a massa do ROV e $h$ representa os efeitos hidrodinâmicos desconhecidos, aqui considerados na forma de amortecimento quadrático, i.e, $h=c \dot{z}|\dot{z}|$ onde $c$ é o coeficiente de amortecimento. Considerando também que devido a baixa velocidade operacional dos ROVs, a massa $m$ não pode ser conhecida com exatidão por conta dos efeitos hidrodinâmicos, podemos atribuir a esse termo uma estimativa e uma incerteza associada, ou seja, $m=\hat{m}+$ $\Delta m$. Logo a Eq. (2) pode ser reescrita na seguinte forma:

$$
\hat{m} \ddot{z}=u+d
$$

onde $d=h-\Delta m \ddot{z}$ é o termo de perturbação do sistema.

Utilizando o Método de Linearização por Realimentação para o sistema descrito pela Eq. (3), a lei de controle pode ser expressa na seguinte forma:

$$
u=\hat{m}\left(\ddot{z}_{d}-2 \lambda \dot{e}-\lambda^{2} e\right)-\hat{d}
$$

onde $e=z-z_{d}$ é o erro de rastreamento, $\hat{d}$ é a estimativa da perturbação $\hat{d}$ e $\lambda$ é uma constante estritamente positiva. $\mathrm{O}$ termo $\hat{d}$ pode ser calculado com base na lei de adaptação,

$$
\hat{d}_{i+1} \leftarrow \hat{d}_{i}+\eta s \Delta t
$$

onde $\eta$ é a taxa de aprendizagem, $s$ é uma variável inspirada no controlador por modos deslizantes e que pode ser calculada por $s=\dot{e}+\lambda e$ e $\Delta t$ é o passo de tempo do controlador.

Se a função $\hat{d}$ aproxima com um erro máximo $\epsilon$, ou seja, $|\tilde{d}| \leq \varepsilon$, onde $\tilde{d}=d-\hat{d}$, então é possível provar que a lei de controle, Eq. (4), delimita o erro de rastreamento dentro de uma região fechada $E=\left\{(e, \dot{e}) \in \mathbb{R}^{2}|| e^{(i)} \mid \leq(i+\right.$ 1)! $\left.\lambda^{i-2} \varepsilon, i=0,1\right\}$ (Bessa et al., 2017).

\section{3. $\epsilon$-GREEDY}

O problema do bandido de vários braços foi introduzido por Robbins (1952) e ele consiste num agente que está lidando repetidamente com $k$ ações diferentes e $N$ ações 
possíveis $A$ nas quais cada ação escolhida tem uma recompensa específica $r_{t}$ (determinística ou não).

O problema consiste em encontrar quais ações levam a uma maior recompensa de forma a maximizar sua recompensa total $\sum_{i=1}^{N} R_{i}$ (para um problema de maximização). Existe um parâmetro neste algoritmo que pode ser mudado de forma a modificar o quanto o agente se torna explorador ou prospectador, chamado $\epsilon$.

Se o valor de $\epsilon$ aumentar (mais explorador), então a recompensa média vai ser inicialmente baixa, mas se torna maior à maneira que o agente descobre as melhores ações e as prospecta. Entretanto, a longo prazo devido a sua natureza exploradora, ele não vai se manter na ação ótima o quanto desejado. Por outro lado, se o valor de $\epsilon$ diminuir (mais prospectador), então a recompensa média vai aumentar mais lentamente, porém é a coisa certa a se fazer para maximizar a recompensa esperada no próximo passo. Entretanto, diferente do caso explorador, ao longo prazo ele irá escolher o caso ótimo mais comumente.

Nós fazemos a suposição de considerar uma distribuição de Bernoulli na qual a ação pode ter recompensa 0 ou 1. Portanto, o problema é apenas analisar a recompensa média dado o tempo $T$ (ou o passo $P$ ). Considere que $Q=\frac{1}{N} \sum_{i=1}^{N} R_{i}$ é a soma das recompensas médias e $R_{i}$ é a recompensa da $i$-ésima ação e a melhor recompensa é chamada de $\mu_{i}$. A diferença entre $\Delta_{i}=\mu_{i}-R_{i}$ é chamada de "erro". então $\Omega=\frac{1}{N} \sum_{i=1}^{N}\left(\mu_{i}-Q\right)$. O nosso objetivo é reduzir o $\Omega$ e para isso, o valor $\epsilon$ determina a maneira na qual o algoritmo chega no valor final $\Omega$ e o quão rápido ele o atinge.

\section{DESENVOLVIMENTO}

\subsection{Controle}

A implementação do controlador pode ser feita de acordo com o Algoritmo 1.

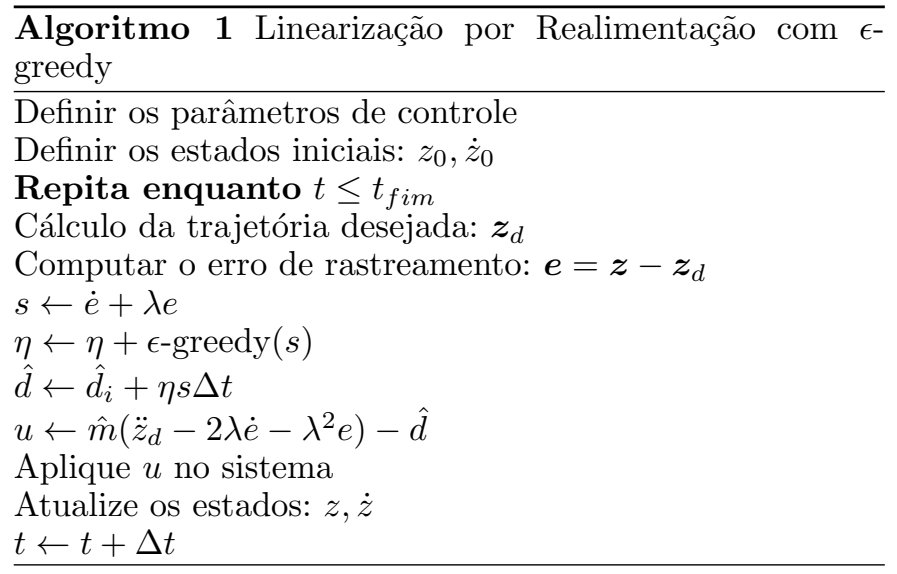

\subsection{Implementando o $\epsilon$-greedy}

No algoritmo $\epsilon$-greedy, $R_{i}$ significa a recompensa após a $i$ ésima ação escolhida, $q(a) \doteq \mathbb{E}\left[R_{t} \mid A_{t}=a\right]$ é o valor de uma ação arbitrária $a$ dada uma recompensa $R_{t}$ e $Q_{n}$ é o valor estimado da ação depois que ela foi escolhida, calculada com base na média das $R_{i}$ recompensas.
Aplicando para o nosso problema, existem três ações possíveis, que seriam incrementar, manter ou diminuir o valor da variável $\eta$ do compensador.

O conceito de recompensa $R$ para o nosso problema seria a medida do erro combinado, $s=\dot{e}+\lambda e$, considerando que se trata de um problema de minimização, quanto menor for o valor deste $s$ melhor. No Algoritmo 2 é mostrado como a implementação dessa técnica é realizada.

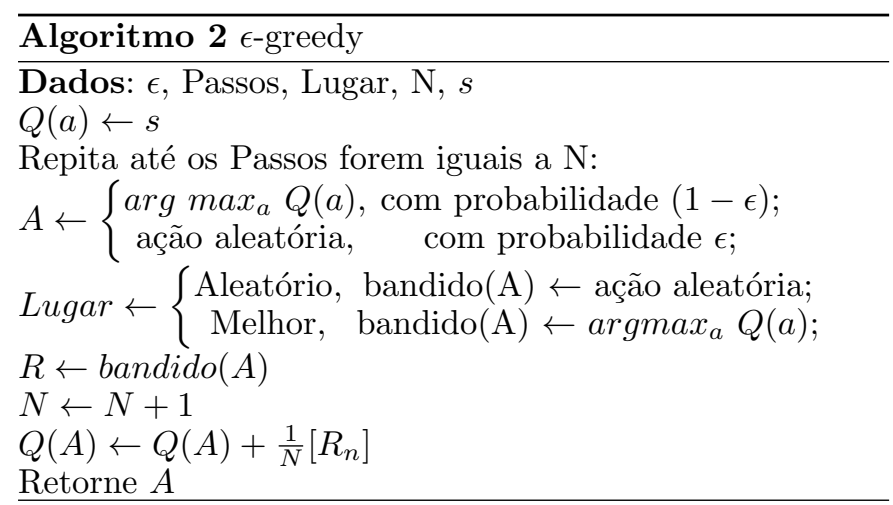

Seguindo os Algoritmos 1 e 2, é possível sintetizar o controlador proposto no esquema mostrado pela Fig. 1.

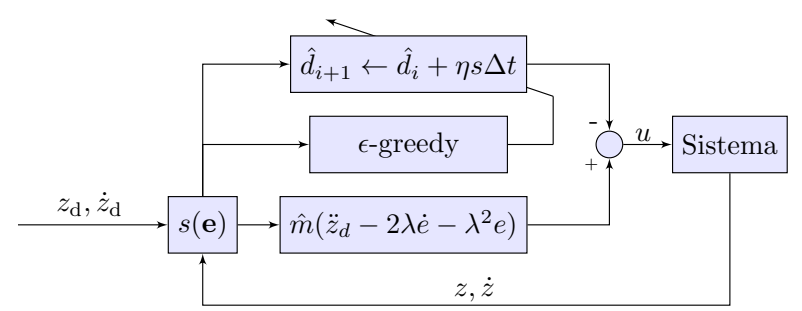

Figura 1. Esquema do controlador.

\section{RESULTADOS NUMÉRICOS}

O sistema de controle proposto foi implementado por meio de simulações. O método de Runge-Kutta de quarta ordem foi utilizado e as taxas de amostragem do controlador foram de $100 \mathrm{~Hz}$ e para o simulador, $1000 \mathrm{~Hz}$ para o sistema dinâmico que foi assumido. No modelo dinâmico foi considerado que $m=50 \mathrm{~kg}$ e $c=250 \mathrm{~kg} / \mathrm{m}$. No controlador foi considerado um erro de $10 \%$ na massa, que os efeitos hidrodinâmicos são desconhecidos, que o parâmetro $\lambda=1$ e $\hat{d}_{0}=0$. Foi considerado também que o atuador do ROV poderia fornecer um esforço de controle limitado em $u_{\max }= \pm 20 \mathrm{~N}$. A trajetória desejada é dada por $z_{d}=0.5(1-\cos (0.1 \pi t))$.

Utilizando os Algoritmos 1 e 2, pode-se encontrar diferentes valores de $\eta$ que induzissem menor erro combinado médio $\bar{s}$, Fig. 2. Para $\epsilon=0.6$, foi encontrado um valor de taxa de aprendizagem ótima de $\eta_{o t}=333$.

Após treinar o modelo com o $\epsilon$-greedy, os resultados mostram que a estratégia de controle com uma otimização do parâmetro adaptativo possui resultados satisfatórios para o rastreamento, Fig. 3 . 


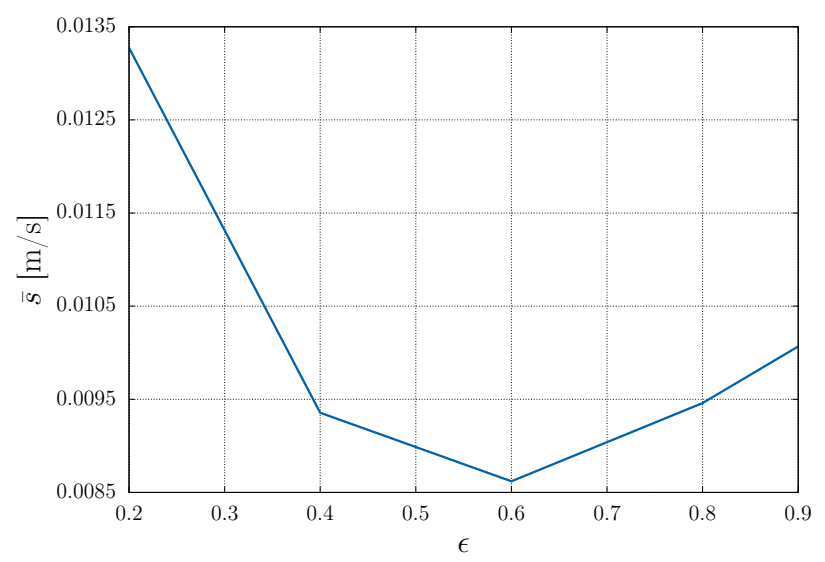

Figura 2. Variação no erro combinado médio $\bar{s}$ pela mudança de $\epsilon$ do $\epsilon$-greedy.

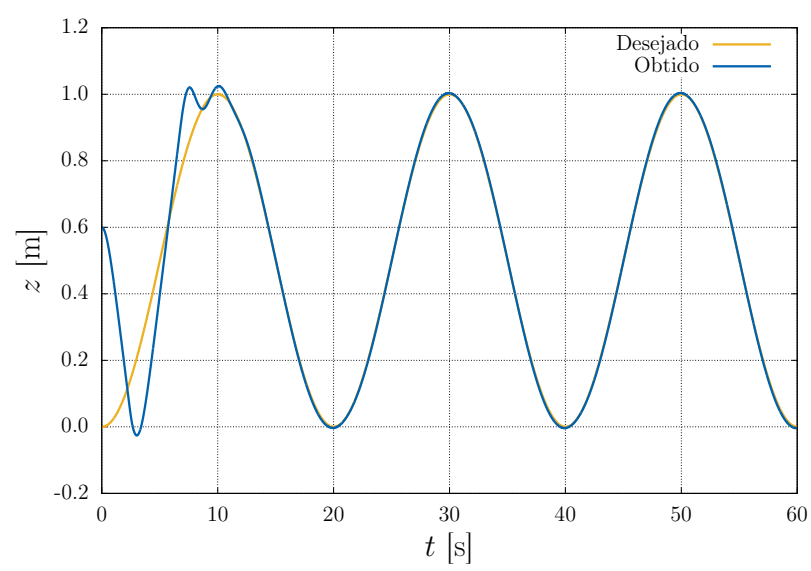

Figura 3. Rastreamento de trajetória para $\eta=\eta_{o t}$.

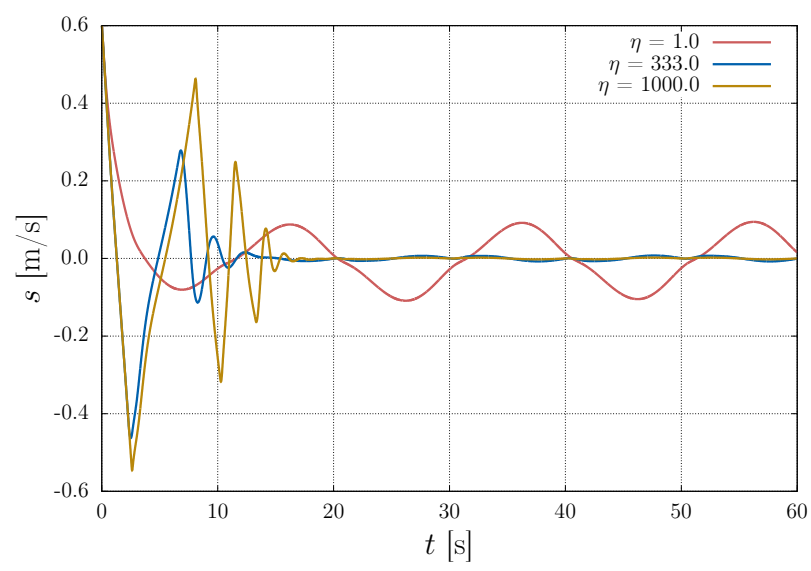

Figura 4. Erro combinado $s$ para diferentes valores de $\eta$.

Testando valores de $\eta$ distintos do $\eta_{o t}$, pode-se notar que valores baixos de $\eta$ dificultam o rastreamento, enquanto valores altos conduzem a uma instabilidade no regime transitório. Isso pode ser melhor visualizado através do erro combinado $s$ na Fig. 4.

Outra análise que pode-se fazer é que com a utilização do otimizador do parâmetro adaptativo, houve também uma diminuição na saturação do esforço de controle necessário em relação ao $\eta=1000$, portanto, podemos observar a

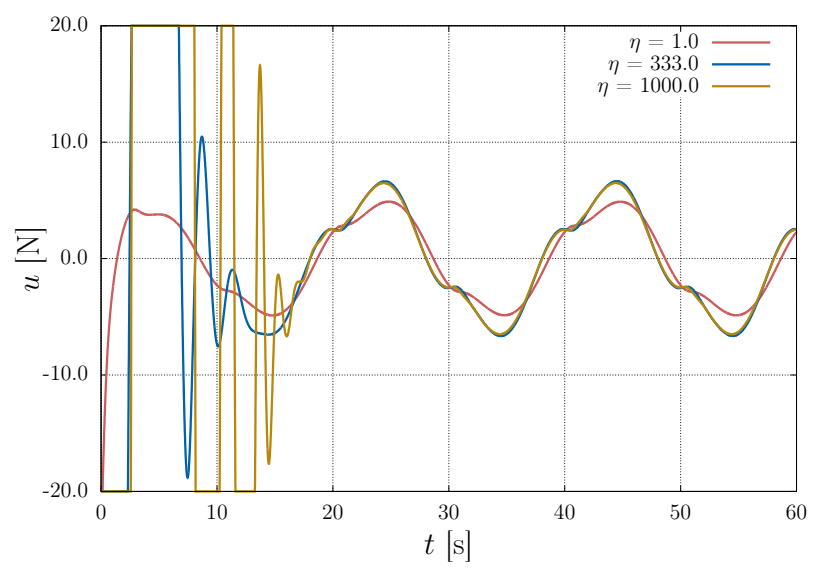

Figura 5. Esforço de controle para diferentes valores de $\eta$.

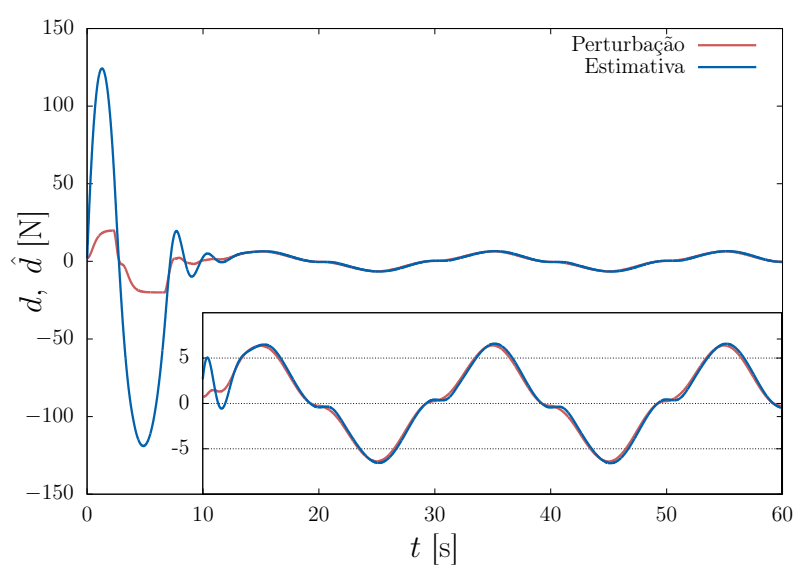

Figura 6. Perturbação do sistema e sua estimativa usando $\eta_{o t}$.

necessidade de otimização deste parâmetro com o objetivo de reduzir o consumo de energia do sistema, Fig. 5.

Pode-se visualizar pela Fig. 6, a estimativa da perturbação usando a lei de adaptação, 5. Nota-se que após um período inicial de adaptação, foi possível estimar com boa precisão a dinâmica não modelada do sistema.

\section{CONCLUSÕES}

Neste artigo, a linearização por realimentação foi combinada com um algoritmo de aprendizagem por reforço, o $\epsilon$ greedy, para lidar com o problema de rastreamento de trajetória para um ROV. Por meio de simulações numéricas, pôde-se observar a melhora na performance do esquema proposto sobre o controlador utilizando a linearização por realimentação tradicional. Para o problema proposto, é visto que a utilização do $\epsilon$-greedy para otimização do parâmetro adaptativo $\eta$ pode resolver o problema com grande performance.

\section{AGRADECIMENTOS}

Os autores agradecem a Coordenação de Aperfeiçoamento de Pessoal de Ensino Superior (CAPES) e ao Conselho Nacional de Desenvolvimento Científico e Tecnológico $(\mathrm{CNPq})$. 


\section{REFERÊNCIAS}

Bejar, E. and Moran, A. (2019). Control of a twodimensional magnetic positioning system with deep reinforcement learning and feedback linearization. Midwest Symposium on Circuits and Systems, (1), 909-912. doi: 10.1109/MWSCAS.2018.8623948.

Bessa, W.M., Dutra, M.S., and Kreuzer, E. (2008). Depth control of remotely operated underwater vehicles using an adaptive fuzzy sliding mode controller. Robotics and Autonomous Systems, 56(8), 670-677. doi:10.1016/j. robot.2007.11.004.

Bessa, W.M., Dutra, M.S., and Kreuzer, E. (2010). An adaptive fuzzy sliding mode controller for remotely operated underwater vehicles. Robotics and Autonomous Systems, 58, 16-26.

Bessa, W.M., Kreuzer, E., Lange, J., Pick, M.A., and Solowjow, E. (2017). Design and adaptive depth control of a micro diving agent. IEEE Robotics and Automation Letters, 2(4), 1871-1877.

Brinkmann, G., Bessa, W.M., Duecker, D.A., Kreuzer, E., and Solowjow, E. (2018). Reinforcement learning of depth stabilization with a micro diving agent. Proceedings - IEEE International Conference on Robotics and Automation, V, 6197-6203. doi:10.1109/ICRA.2018. 8461137.

Goheen, K.R. and Jeffreys, E.R. (1990). Multivariable self-tuning autopilots for autonomous and remotely operated underwater vehicles. IEEE Journal of Oceanic Engineering, 15(3), 144-151.

Haykin, S. (2007). Redes neurais: princípios e prática. Bookman Editora.

Jardine, P.T., Kogan, M., Givigi, S.N., and Yousefi, S. (2019). Adaptive predictive control of a differential drive robot tuned with reinforcement learning. International Journal of Adaptive Control and Signal Processing, 33(2), 410-423. doi:10.1002/acs.2882.

Kim, J.B., Kwon, D.H., Hong, Y.G., Lim, H.K., Kim, M.S., and Han, Y.H. (2019). Deep Q-Network Based Rotary Inverted Pendulum System and Its Monitoring on the EdgeX Platform. 1st International Conference on Artificial Intelligence in Information and Communication, ICAIIC 2019, 34-39. doi:10.1109/ICAIIC.2019. 8668979.

Ludvigsen, M. and S $\phi$ rensen, A.J. (2016). Towards integrated autonomous underwater operations for ocean mapping and monitoring. Annu. Rev. Control, 42, 145157.

Mindell, D.A. (2015). Ourselves: Robotics and the Myths of Autonomy. Viking.

Pradeep, D.J., Noel, M.M., and Arun, N. (2016). Nonlinear control of a boost converter using a robust regression based reinforcement learning algorithm. Engineering Applications of Artificial Intelligence, 52, 1-9. doi:10. 1016/j.engappai.2016.02.007.

Robbins, H. (1952). Some aspects of the sequential design of experiments. Bulletin of the American Mathematical Society, 58(5), 527-535.

Teague, J., Allen, M.J., and Scott, T.S. (2018). The potential of low-cost ROV for use in deep-sea mineral, ore prospecting and monitoring. Ocean. Eng., 147, 333339.

Yang, X., He, H., Wei, Q., and Luo, B. (2018). Reinforcement learning for robust adaptive control of parti- ally unknown nonlinear systems subject to unmatched uncertainties. Information Sciences, 463-464, 307-322. doi:10.1016/j.ins.2018.06.022.

Yuh, J. (1994). Learning control for underwater robotic vehicles. IEEE Control Systems Magazine, 14(2), 3946.

Zhu, L.M., Modares, H., Peen, G.O., Lewis, F.L., and Yue, B. (2015). Adaptive suboptimal output-feedback control for linear systems using integral reinforcement learning. IEEE Transactions on Control Systems Technology, 23(1), 264-273. doi:10.1109/TCST.2014.2322778. 